\title{
Ways to reduce the error of additive methods of forming
}

\author{
Alexander N. Grechukhin*, Vadim V. Kuts, and Michail S. Razumov \\ Southwest State University, 305040 Kursk u.50 Let Oktyabrya 94, Russian Federation
}

\begin{abstract}
The article is devoted to the investigation of the surface layer formation accuracy of engineering products by additive methods. The analysis of advantages and disadvantages of layered products synthesis technologies was carried out. It was revealed that, in additive shaping, the exact characteristics of the surface layer differ significantly from the accuracy characteristics of the surface layer of products obtained by the traditional methods. The analysis of domestic and foreign works on the topic of research was carried out. It is revealed that to increase the accuracy characteristics of products obtained by additive methods, it is necessary to realize dynamically the spatial orientation of the working element of the additive installation in the process of shaping. To control the spatial orientation of the working organ of additive equipment, a method is proposed. The proposed methodology will allow to calculate the controlled parameters of the process equipment, to provide the required orientation of the working element of the additive installation to reduce the error of shaping (approximation).
\end{abstract}

\section{Introduction}

Formation of surfaces of engineering parts is the central task, solved in the production process. Currently, there is a steady tendency towards the increase in the complexity of products, more stringent requirements to the terms of launching new products, so the development of new methods for forming products becomes relevant.

According to the classification of forming methods, proposed by Burns [1], there are the following methods of forming: subtractive, formative and additive.

The subtractive method of forming involves shaping the part by removing some material from the initial workpiece.

The formative method of forming involves shaping the part by affecting the initial workpiece or material of the tooling elements without removing material from the surface.

The additive method is a method of forming, based on the layer-by-layer addition of material. Additive methods include layer-by-layer synthesis, in which the material is added in the form of flat or concentric layers. Layer-by-layer synthesis has the following features: the product surfaces are formed by the sides of the added layers, approximating the shape

\footnotetext{
*Corresponding author: agrechuhin@mail.ru
} 
of the product; the accuracy of the formed products is limited by the minimum layer thickness [2].

The scope of application of the layer-by-layer synthesis technologies remains limited in comparison with subtractive and formative technologies due to the following factors: low efficiency of the process of product manufacturing, its high energy consumption, and the high cost of processing equipment, as well as raw materials, low accuracy of formed products.

\section{Analysis of domain research}

A large number of scientific papers consider the mechanisms of error formation during forming with subtractive methods.

The paper [3] studies the problems of error formation during machine processing; the processing error was considered as a result of the action of the corresponding components:

$$
\Delta r_{0}=\Delta r_{s t}+\Delta r_{d y n}+\Delta r_{\text {temp }}+\Delta r_{\text {toolwear }}+\Delta r_{\text {partswear }}
$$

where $\Delta r_{s t}$ is the static component of the processing error; $\Delta r_{d y n}$ is the dynamic component of the processing error; $\Delta r_{\text {temp }}$ and $\Delta r_{\text {toolwear }}$ are the errors caused by the temperature deformations and wear of the tool, respectively; $\Delta r_{\text {partswear }}$ is the error caused by the wear of parts of the units.

It should be noted that forming of parts using additive methods is characterized by high values of the static component of the processing error, in particular, the value of the forming error (approximation). This is due to the fact that the formation of the surface layers of complex parts occurs line-by-line, while the orientation of the forming element of the additive plant remains constant and independent of the curvature of the surface being formed [4] (Fig. 1).

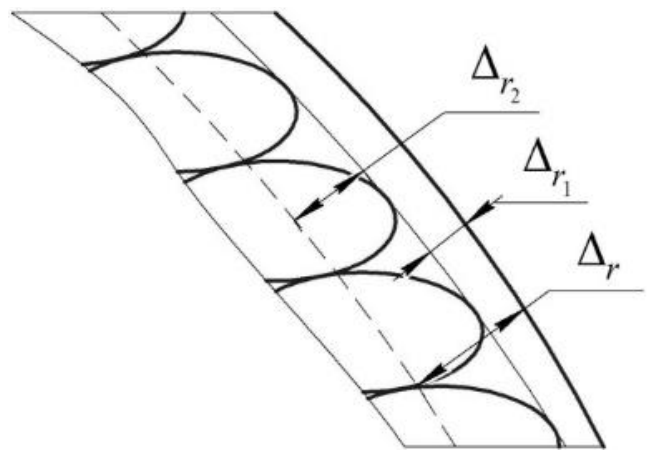

Fig. 1. Scheme for determining the error during forming using additive methods with a constant orientation of the forming element of the additive plant, where $\Delta_{r}$ is the total error, $\Delta_{r_{1}}$ is the error of the shape, $\Delta_{r_{2}}$ is the error of forming (approximation).

The error during forming using additive methods may be considered as the algebraic sum of the error in the shape of the nominal surface of the part and the error in the forming (approximation) of the nominal surface section with layers of the deposited material:

$$
\Delta_{r}=\Delta_{r_{1}}+\Delta_{r_{2}}
$$


where $\Delta_{r_{1}}$ is the error of the shape, $\Delta_{r_{2}}$ is the error of forming (approximation).

The error of the product shape obtained using additive methods depends on the accuracy of the processing equipment and consists of elementary errors caused by the geometric inaccuracy of the additive equipment, thermal deformations, the accuracy of the equipment dimensional settings, and the rigidity of the processing system. In turn, the error of forming (approximation) depends on the following parameters: layer thickness, forming temperature, forming speed, material properties, and technological features of the additive equipment.

The accuracy of additive methods of forming is currently insufficiently studied; therefore, the purpose of the study is the development of new theoretical background, which in the future can become a basis for upgrading the existing equipment and construction of new equipment for additive manufacturing.

One of the ways to solve the problem of reducing the error of forming (approximation) of additive methods is to reduce the curvature of the applied elementary layer surface at the point of contact with the nominal surface of the part (Fig. 2) [4, 5].

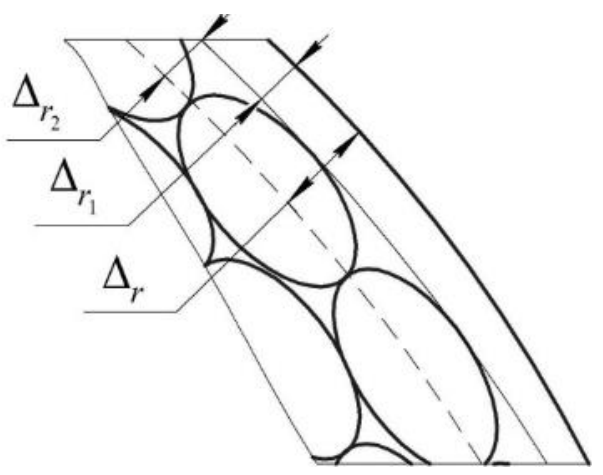

Fig. 2. Formation of the error during forming using additive methods with the orientation of the forming element of the additive plant relative to the surface being obtained; where $\Delta_{r}$ is the total error, $\Delta_{r_{1}}$ is the error of the shape, $\Delta_{r_{2}}$ is the error of forming (approximation).

\section{Construction of the model of forming system of additive equipment}

One of the solutions to this problem is to provide a spatial orientation of the working element of the additive equipment along the normal line at the point of the nominal surface of the formed part, for which it is necessary to develop new additive equipment or to expand the technological capabilities of the existing additive equipment.

A review of Russian and foreign literature shows that a large number of scientific papers consider the problems related to the provision of the spatial orientation of the working element of the additive plant in the process of forming [6-14]. However, these papers consider the provision of the static orientation, and the problems related to the dynamic orientation of the forming element of the additive equipment in the process of forming are not considered.

To provide the spatial orientation of the forming element of the additive equipment in the process of forming, it is necessary to solve the problem of calculating the controlled parameters of the processing equipment, at which the necessary position of the forming element will be obtained. 
In this regard, the basic equation of the additive forming process with the use of processing equipment that provides the spatial orientation of the forming element of the additive plant is described. The equation is as follows:

$$
\bar{r}_{0}(u, v)=A_{0 \Sigma} \cdot A_{\Sigma}\left(q_{1}, \ldots, q_{m}\right) \cdot e^{-4}
$$

where $A_{0 \Sigma}$ is the matrix for setting the coordinate system of the part in the working space of the processing equipment; $A_{\Sigma}\left(q_{1}, \ldots, q_{m}\right)$ is the matrix of the forming system of the processing equipment, which corresponds to its parameters $q_{1}, \ldots, q_{m} ; e^{-4}$ is the radius vector of the origin of coordinates; $u, v$ are the curvilinear surface coordinates.

The parameters $q_{1}, \ldots, q_{m}$, included in the matrix $A_{\Sigma}$, can be divided into controlled and uncontrolled (constructive) parameters of the processing equipment.

It is necessary to apply the following relationship to the uncontrolled parameters of the processing equipment in order to ensure the process of forming,

$$
q_{i}=\text { const } .
$$

It is necessary to apply the following relationship to the controlled parameters in order to ensure the process of forming,

$$
q_{i}=q_{i}(u, v)
$$

When the $j$ point of the part surface is formed, equation (5) can be determined by solving the matrix equation (Fig. 3):

$$
A_{0 j}\left(u_{j}, v_{j}\right) \cdot A_{0 \Sigma} \cdot A_{\Sigma}\left(q_{1}, \ldots, q_{n}\right)=E,
$$

where $A_{0 j}\left(u_{j} ; v_{j}\right)$ is the matrix of the transfer from the coordinate system of the formed part to the coordinate system of the $j$ point on the part surface $\bar{r}_{0 j}\left(u_{j}, v_{j}\right) ; E$ is the identity matrix.

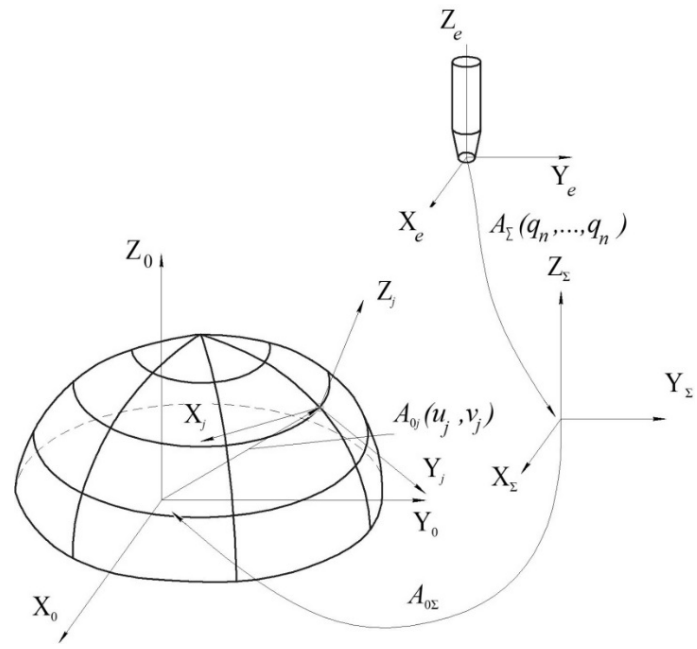

Fig. 3. Scheme for calculating controlled parameters to provide the spatial orientation of the additive plant extruder. 
The matrix $A_{0 j}$ of the transition from the coordinate system of the formed part to the coordinate system of the $j$ point on the part surface $\bar{r}_{0 j}\left(u_{j}, v_{j}\right)$ is calculated according to the method described in [15-20], using the vectors defining the positive direction of the axis $Z_{j} \quad \bar{k}_{j 0}=\frac{\partial \bar{r}_{o j}}{\partial u} \times \frac{\partial \bar{r}_{o j}}{\partial v} /\left|\frac{\partial \bar{r}_{o j}}{\partial u} \times \frac{\partial \bar{r}_{o j}}{\partial v}\right|$, axis $Y_{j}, \bar{l}_{j 0}=\frac{\partial \bar{r}_{o j}}{\partial u} /\left|\frac{\partial \bar{r}_{o j}}{\partial u}\right|, \bar{l}_{j 0}=\frac{\partial \bar{r}_{o j}}{\partial v} /\left|\frac{\partial \bar{r}_{o j}}{\partial v}\right|$, where $\frac{\partial \bar{r}_{o j}}{\partial u}$, $\frac{\partial \bar{r}_{o j}}{\partial v}$ differential derivatives of the vector-function with respect to the parameters $u, v$, as well as vector $\bar{r}_{o j}$, specifying the position of the origin of the coordinate system $X_{j} Y_{j} Z_{j}$.

\section{Conclusions}

The proposed methodology will allow calculating the controlled parameters of the processing equipment, providing the required orientation of the forming element of the additive plant to reduce the error of forming (approximation) using 5-axis and 6-axis positioning mechanisms.

The study was performed as part of the state order No. 2.9320.2017/8.9

\section{References}

1. M. Burns, Automated Fabrication: Improving Productivity in Manufacturing (PTR Prentice Hall, USA, 1993)

2. A. A. Saprykin, Increasing the productivity of the process of selective laser sintering in the manufacture of prototypes. Thesis of candidate of technical Sciences (Tomsk Polytechnic University, Yurga, 2006)

3. A. Pronikov, O. I. Averyanov, Yu. S. Apollo, Designing of metal-cutting machines and machine tools, 3 (MSTU them NE Bauman: Mechanical Engineering, Moscow, 1994)

4. V.V. Kuts, M.S. Razumov, A.N. Grechukhin, N.A. Bychkova, Int. J. Appl. Eng. Res., 11 (24), 11832 (2016)

5. L. M. Chervyakov, N. A. Bychkova, N. V. Yeliseyeva and others, Transfer of additive technologies: the industry of the Kursk region (ZAO «University Book», Kursk, 2016)

6. V. L. Dobroskok, L. N. Abdurayimov, S. I. Chernyshov, Scientific notes of the Crimean Engineering and Pedagogical University, 24, 13 (2010)

7. S. K. Singhal, A. P. Pandey, P. M. Pandey, A. K. Nagpal, Computer-Aided Design and Applications, 2 (1-4), 319 (2005)

8. H. S. Hong, K. H. Lee, Int. J. Adv. Manuf. Technol., 28 (3-4), 307 (2006)

9. H. S. Hong, K. H. Lee, Proc. of the 34th International Conference on Computers \& Industrial Engineering, 426 (2004)

10. J. Hur, K. Lee, Manuf. Technol., 14 (4), 247 (1998)

11. J. Y. Kim, K. Lee, J. C. Park, Technical Report. Dept Mech Des Prod Eng (Seoul National University, Seoul), 356 (1994)

12. P. T. Lan, S. Chou, Y. Chent, L. D. Gemmill, Computer-Aided Design, 29 (1), 53 (1997)

13. S.H. Massod, W. Rattanawong, P. Iovenitti, J. Mater. Process. Technol, 139, 110 (2003) 
14. S. H. Masood, W. Rattanawong, Int. J. Adv. Manuf. Technol., 19, 209 (2002)

15. I. N. Egorov, Position-force control of robotic and mechatronic devices (Vladimir State University, Vladimir, 2010)

16. S. I. Lashnev, A. N. Borisov, S. G. Emelyanov, Geometric theory of surface formation by cutting tools (Kursk. state. tech. un-t., Kursk, 1997)

17. S. G. Emelyanov, Development of the theory. methods and means of surface formation by assembled metal-cutting tools on the basis of system modeling of their design process Thesis of doctor of technical Sciences (MGTU, Moscow, 2001)

18. V. V. Kuts, Methodology of pre-project studies of specialized metal-cutting systems Thesis of doctor of technical Sciences (Kursk State Technical University, Kursk, 2012)

19. V. V. Kuts, V. V. Ponomarev, Fundamental and applied problems of technology, 2(322), 110 (2017)

20. V. A. Grechishnikov, V. V. Kuts, M. S. Razumov, V. B. Romanov, P.M. Pivkin, A.N. Grechikhin, S. Yu. Yurasov, STIN, 4, 24 (2017)

21. V. A. Grechishnikov, V. B. Romanov, P. M. Pivkin, Russian Engineering Research, 37 (9), 824 (2017)

22. High-precision industrial 6-coordinate robot, access mode: //video.phim22.com/n535u234a405i3w5m5b3x5.html 